\title{
Statistical patterns of deformation localization during plastic flow in the AMg6 alloy
}

\author{
D. V. Efremov ${ }^{\dagger, 1}$, S. V. Uvarov ${ }^{1}$, L. V. Spivak², O. B. Naimark ${ }^{1}$ \\ †efremov.d@icmm.ru \\ ${ }^{1}$ Institute of Continuous Media Mechanics UB RAS, 1 Academician Korolev St., Perm, 614013, Russia \\ ${ }^{2}$ Perm State University, 15 Bukireva St., Perm, 614990, Russia
}

\begin{abstract}
The laws governing the development of localization of plastic deformation under the conditions of manifestation of the effect of space-time heterogeneity (the Portevin-Le Chatelier effect) were investigated. Compression tests were carried out on cylindrical specimens of the aluminum alloy AMg6 with a constant strain rate of $0.4-1.7 \cdot 10^{-1} \mathrm{~s}^{-1}$, inclined by $2^{\circ}$ from the vertical. This shape of the specimens determines a preferential direction for the formation of slip bands that allows for achieving large strains (up to 80\%) without failure. Calorimetric studies of samples of the AMg6 alloy with varying degrees of deformation were carried out using a STA "Jupiter" 449 calorimeter. Statistical distributions of the intervals between the plastic flow stress fluctuations were analyzed and two critical points were found that indicate a change in the mechanisms of plastic deformation. The transition through the first critical point corresponds to the formation of multiple regions of localized plasticity. The further plastic flow of the studied alloy reveals multiscale signs of localization of plastic deformation over the rest of the plastic flow curve. At the stage of developed plastic flow, the maximum level of stored energy is observed during deformation. The transition through the second critical point is associated with the formation of nuclei of macroscopic failure, which is accompanied by the release of the stored strain energy and a decrease in calorimetric effects. The form of the probability density function of the intervals between the plastic flow stress fluctuations and the probability density function of the flow stress fluctuations confirm the self-similar nature of multiscale correlations of these quantities, similar to that observed for a closed turbulent flow of fluids between two rotating disks in the Karman experiment with large Reynolds numbers.
\end{abstract}

Keywords: strain localization, critical points, calorimetry.

УДК: 548.736 .12

\section{Статистические закономерности развития локализации деформации при пластическом течении в сплаве АМг6}

\footnotetext{
Ефремов Д. В. ${ }^{\dagger, 1}$, Уваров С. В. ${ }^{1}$, Спивак Л. В. ${ }^{2}$, Наймарк О. Б. ${ }^{1}$

${ }^{1}$ Институт механики сплошных сред УрО РАН, ул. Академика Королёва, 1, Пермь, 614013, Россия

${ }^{2}$ Пермский государственный национальный исследовательский университет, ул. Букирева, 15, Пермь, 614990, Россия

Исследованы закономерности развития локализации пластической деформации в условиях проявления эффекта пространственно-временной неоднородности (эффект Портевена-Ле Шателье). Испытания по осадке проведены на наклоненных на $2^{\circ}$ от вертикали цилиндрических образцах из алюминиевого сплава АМг6 с постоянной скоростью деформации $0.4-1.7 \cdot 10^{-1} \mathrm{c}^{-1}$ на электромеханической машине Shimadzu AG-300. Такая форма образцов создаёт преимущественное направление образования полос скольжения, что позволяет достичь больших деформаций (до 80\%) без разрушения образца. Проведены калориметрические исследования образцов из сплава АМг6 с различной степенью деформации с помощью прибора STA «Jupiter» 449. Проведён анализ статистических распределений интервалов следования флуктуаций напряжения пластического течения, установлено существование двух критических точек, которые свидетельствуют о смене механизмов локализации пластической деформации. Переход через первую критическую точку соответствует формированию множественных областей локализованной пластичности. Дальнейшее пластическое течение исследованного сплава обнаруживает многомасштабные признаки локализации пластической деформации на всём оставшемся протяжении кривой пластического течения. На стадии
} 
развитой пластической текучести наблюдается максимальный уровень запасённой энергии в ходе деформации. Переход через вторую критическую точку связан с формированием очагов макроскопического разрушения, что сопровождается высвобождением запасённой энергии деформирования и уменьшением калориметрических эффектов. Вид функции плотности вероятности флуктуаций времени ожидания между событиями и флуктуаций напряжения пластического течения подтверждают автомодельный характер многомасштабных корреляций данных величин, аналогичный наблюдаемому для замкнутого турбулентного течения жидкостей между двумя вращающимися дисками в опыте Кармана при больших числах Рейнольдса.

Ключевые слова: локализация деформации, критические точки, калориметрия.

\section{1. Введение}

Исследования посвящены изучению пространственновременной динамики деформации в сплаве АМг6 в условиях формирования множественных областей локализованного сдвига. Анализ статистических распределений интервалов следования флуктуаций напряжений пластического течения установил существование двух критических точек, переходы через которые сопровождается качественной сменой механизмов локализации пластической деформации. Термодинамические закономерности развития пластического течения изучаются методом дифференциальной сканирующей калориметрии с последующим вычислением запасённой энергии для образцов с разной степенью деформации. Установлена связь калориметрических эффектов со сменой сценариев развития пластической деформации, характерных для неравновесных критических систем.

Ранее в работах [1-3] исследована локализация пластической деформации, обусловленная коллективным поведением дефектов. В [4-7] установлено, что пластическая деформация твёрдых тел на протяжении всего процесса пластического течения имеет локализованный на макроскопическом масштабном уровне характер. При этом деформирование реализуется в форме лавиноподобных сдвигов, размеры которых подчиняются степенной статистике. Данные закономерности соответствуют результатам опубликованных в [6,7], где исследованы статистические закономерности формирования поверхностного профиля пластически деформированных поликристаллических образцов меди.

В работе [5] выделены стадии процесса деформирования при сопоставлении с данными о распределении зон локализации пластического течения, что позволило установить универсальный сценарий коррелированного поведения многомасштабных зон локализации пластической деформации, практически не зависящий от конкретных деталей механизма деформации и структуры деформируемого материала.

К основным закономерностям множественной локализации пластического течения могут быть отнесены следующие [5]:

- локализация возникает самопроизвольно при постоянной скорости растяжения образцов;

- картины локализации последовательно меняются по мере развития пластического течения закономерным образом, а их эволюция тесно связана со стадийностью течения;
- на некоторых стадиях картины локализации обладают отчётливо выраженной пространственной и временной периодичностью;

- каждая из картин локализации связаны с определённым микроскопическим механизмом деформационного упрочнения, действующим на этой стадии;

- дефектная структура материала и деформационное упрочнение необратимо изменяются при пластической деформации, инициируя нелинейный характер развития макроскопического пластического деформирования.

\section{2. Универсальность статистики флуктуаций при неустойчивом пластическом течении металлов}

Примером локализованного пластического течения на стадии упрочнения материала является эффект Портевена-Ле Шателье или прерывистой текучести материала, который характерен для широкого класса пластичных материалов при определённых температурно-скоростных условиях нагружения и выражается на кривых деформирования в виде образования множественных пульсаций напряжения пластического течения, вызванных распространением полос локализованного сдвига [8-11].

Эксперимент на квазистатическое одноосное нагружение (с постоянной скоростью деформации $0.4-1.7 \cdot 10^{-1} \mathrm{~s}^{-1}$ ) был проведён для цилиндрических образцов из алюминиевого сплава АМг6 при комнатной температуре (диаметр 15 мм, высота 22.5 мм и наклоненных на $2^{\circ}$ от вертикали). Данная форма образцов создаёт преимущественное направление образования деформационных полос скольжения и позволяет достичь больших деформаций (до 80\%) без разрушения.

На Рис. 1 представлена деформационная диаграмма алюминиевого сплава, иллюстрирующая множественные флуктуации напряжения течения, обнаруживающие качественно отличную динамику на разных участках деформационной кривой. На Рис. 2 показана поверхность деформированного образца, полученная с помощью электронного микроскопа. На образцах с деформацией 0.35 и 0.69 наблюдается рельеф, вызванный образованием полос скольжения [6,7]. На образце с деформацией 0.69 видны макроскопические трещины разрушения.

Временная последовательность флуктуаций между событиями представлена Рис. 3 и демонстрирует резкое увеличение характерных времен "релаксации» (интервалов следования импульсов) в окрестностях двух значений макроскопической деформации. 


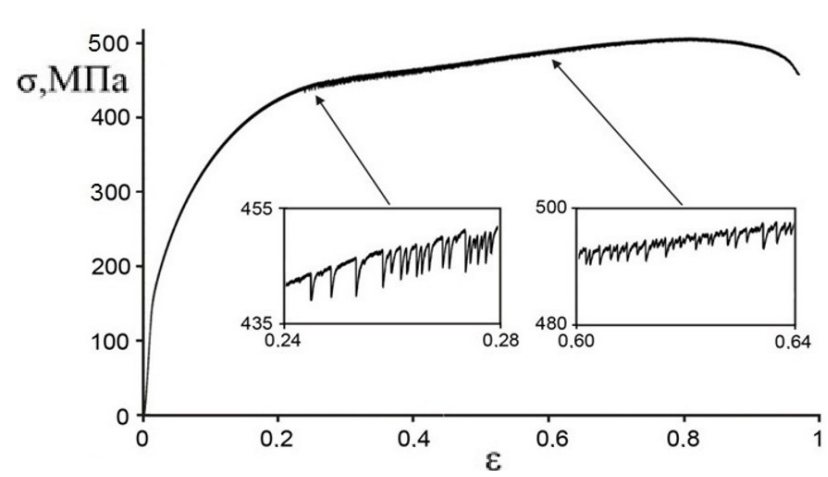

Рис. 1. Деформационная диаграмма алюминиевого сплава АМг6 (скорость деформации $0.8 \cdot 10^{-1} \mathrm{~s}^{-1}$ ).

Fig. 1. Deformation diagram of AMg6 aluminum alloy (strain rate $\left.0.8 \cdot 10^{-1} \mathrm{~s}^{-1}\right)$.

Плотность функции распределения флуктуации интервалов времени между конечно-амплитудными флуктуациями напряжения пластического течения $\sigma$ в переменных $\ln P \sim(\ln t-\langle\ln t\rangle) / D_{t}$ вычисленная для всего участка деформационной диаграммы, представлена на Рис. 4.

Экспериментальное исследование развитого стационарного турбулентного течения (схема Кармана турбулентность в замкнутом объёме, инициированная вращающимися дисками) позволило также установить, что функция плотности вероятности для конечноамплитудных флуктуаций момента может быть представлена с высокой точностью в виде [12,13]:

$$
P(y)=K \exp \left(x(y)-e^{x(y)}\right)^{a},
$$

где $K, b, s-$ эмпирические константы; $x=b(y-s) ; a=\pi / 2$; $y=(\ln t-\langle\ln t\rangle) / D_{t^{*}}$

Аналогично [2] был проведен анализ спектра амплитуд флуктуаций напряжения пластического течения (Рис. 1). Плотность функции распределения флуктуации напряжения пластического течения $\sigma$ в переменных $\ln P \sim(\ln t-\langle\ln t\rangle) / D_{t}$, вычисленная для всего участка деформационной диаграммы, представлена на Рис. 4.

Соответствие зависимостей функции плотности распределения флуктуаций напряжений пластического течения в сплаве и флуктуаций момента при развитом турбулентном течении жидкостей (в условиях резкого

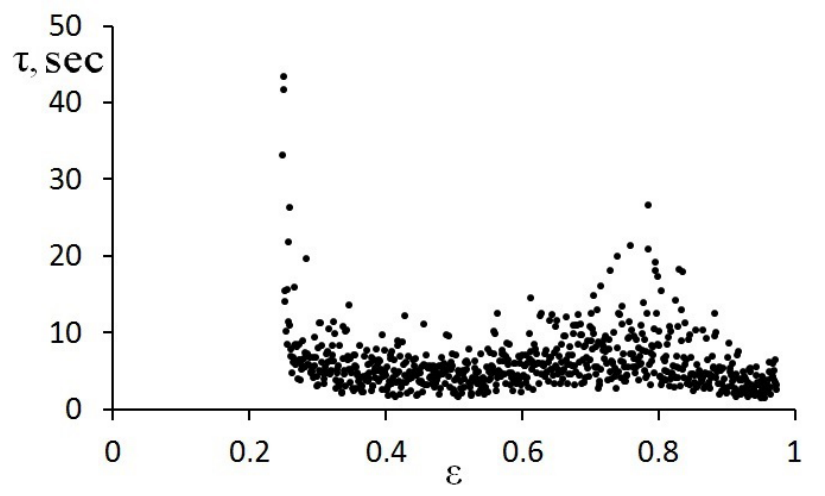

Рис. 3. Зависимость интервалов времени между конечноамплитудными флуктуациями напряжения течения.

Fig. 3. The time dependence between finite amplitude fluctuations of the flow stress.

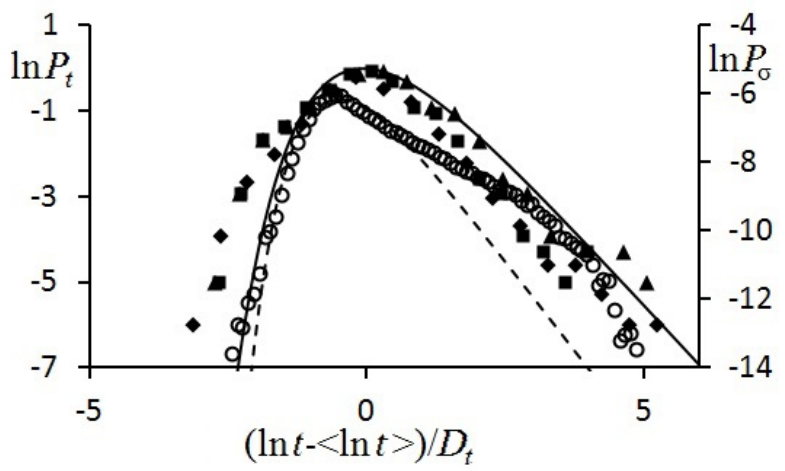

Рис. 4. Плотность распределения флуктуация временных интервалов $\left(P_{t}\right)$ и напряжения $\left(P_{\sigma}\right)$ на стадии пластического течения для сплава АМг6. Экспериментальные данные флуктуации временных интервалов показаны для скорости перемещения захвата: • - 1 мкм/с, - 2 мкм/с, $\Delta-4$ мкм/с; - - расчёт функции плотности вероятности, выполненный по формуле (1), плотность распределения флуктуаций напряжения пластического течения показана: о - экспериментальные данные; - - - расчёт функции плотности вероятности, выполненный по формуле (1).

Fig. 4. The probability density function of time intervals $\left(P_{t}\right)$ and stress $\left(P_{\sigma}\right)$ at the plastic flow stage for AMg6 alloy. Experimental data of time interval fluctuations are shown for the capture movement speed: $\bullet-1 \mu \mathrm{m} / \mathrm{s}, \boldsymbol{-}-2 \mu \mathrm{m} / \mathrm{s}, \boldsymbol{\Delta}-4 \mu \mathrm{m} / \mathrm{s} ;-$ - calculation of the probability density function performed by the equation (1), the distribution density of plastic flow stress fluctuations is shown: $\circ-$ experimental data; - - - the calculation of the probability density function performed by the equation (1).

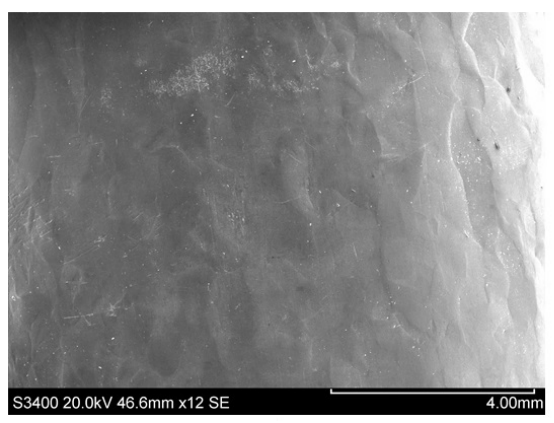

a

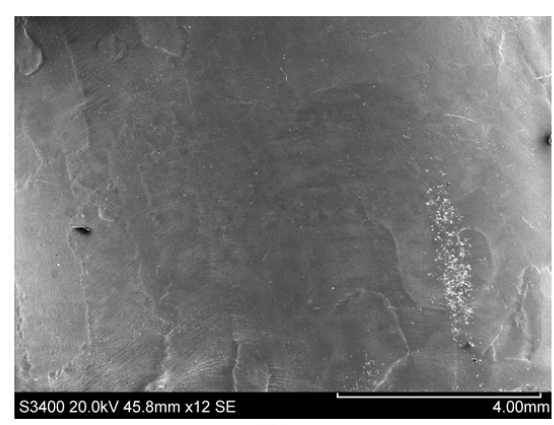

b

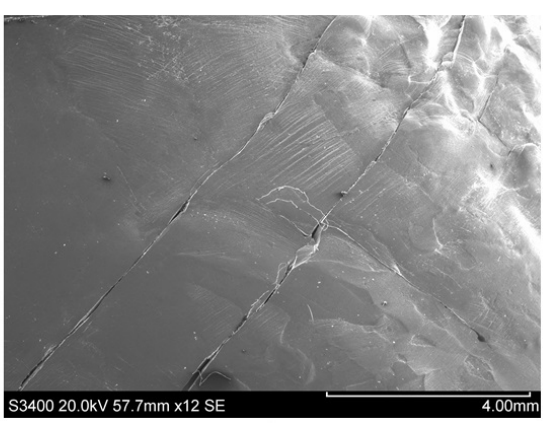

Pис. 2. Поверхность деформированных образцов (увеличение $\times 12$ ): деформация 0.16 (a); деформация 0.35 (b); деформация 0.69 (c). Fig. 2. The surface of a deformed samples (magnification $\times 12$ ): deformation 0.16 (a); deformation 0.35 (b); deformation 0.69 (c). 
падения мощности, инжектируемой в поток), позволяет высказать предположение, что негауссовый характер ветвей функции распределения связан со сценарием, для которого флуктуации амплитуды напряжений пластического течения и флуктуации момента при турбулентном течении могут инициироваться единым механизмом [14].

\section{3. Дифференциальная сканирующая калориметрия}

Для исследования методом дифференциальной сканирующей калориметрии (ДСК) [15,16] была проведена серия дополнительных испытаний по осадке наклоненных цилиндрических образцов из сплава АМг6. Сжатие производилось до значений деформации $0.16 ; 0.35 ; 0.69$ с постоянной скоростью деформации $0.8 \cdot 10^{-1} \mathrm{~s}^{-1}$, после чего эксперимент останавливался и образец извлекался из испытательной машины и подвергался ДСК анализу с помощью прибора STA «Jupiter» 449. ДСК кривые деформированных образцов из сплава АМг6 представлены на Рис. 5.

На ДСК кривых, соответствующих деформированным образцам, в области температур $300^{\circ} \mathrm{C}$ наблюдается экзотермический эффект, который наиболее ярко выражается на образце с деформацией 0.35. Величина запасённой энергии образцов определяется вычислением площади под пиком $[15,16]$ и составляет для деформации: $0.16-0.039$ Дж/г; $0.35-0.525$ Дж/г; $0.69-0.271$ Дж/г. При повторном снятии ДСК кривой для образца с деформацией 0.69, экзотермический эффект в области $300^{\circ} \mathrm{C}$ не наблюдался.

Нагрев пластически деформированного материала инициирует механизм возврата и рекристаллизации, приводящий к появлению новых зёрен. Этим обусловлено высвобождение накопленной при пластической деформации энергии. Для алюминиевых сплавов в области температур $300^{\circ} \mathrm{C}$ наблюдается экзотермическая реакция, связанная с осаждением фазы $\alpha-\mathrm{AlFeSi}[16]$.

\section{4. Заключение}

Получены экспериментальные данные о пространственно-временной локализации пластического течения алюминиевого сплава АМг6 на стадии развитой пластичности в условиях осадки наклонных цилиндрических образцов при разных скоростях перемещения захватов испытательной машины. Проведённый анализ статистических распределений интервалов следования флуктуаций напряжений пластического течения установил существование двух критических точек, которые свидетельствуют о смене механизмов локализации пластической деформации. Вид функции плотности вероятности флуктуации времени ожидания между событиями и флуктуации напряжения пластического течения подтверждает существование возможного подобия механизмов, развития неустойчивостей пластического течения и жидкостей в инерционном интервале развитой турбулентности [14,17-19].

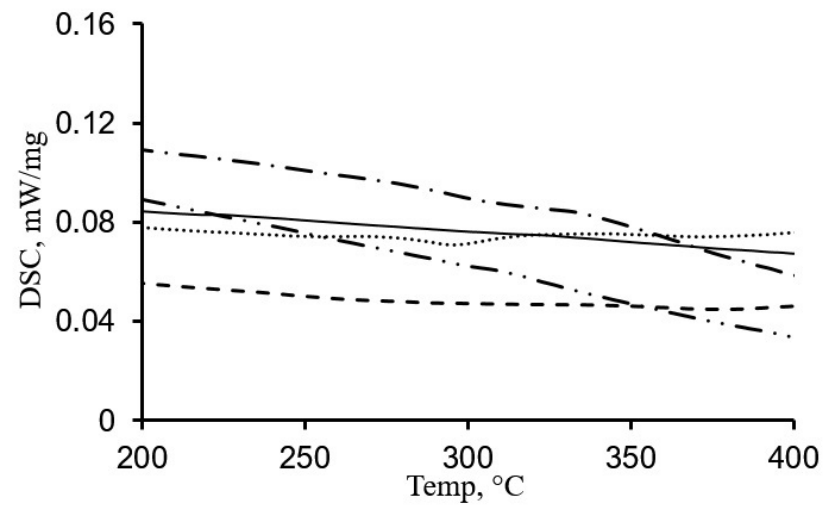

Рис. 5. ДСК кривые для образцов из сплава АМг6 различной степени деформации: --0 ; - -0.16 ; :-0.35; - - 0.69 ; - - - 0.69 (повторное снятие ДСК кривой).

Fig. 5. DSC curves for samples from AMg6 alloy of various degrees of deformation: --0 ; --0.16 ; : - 0.35; - - 0.69; - - 0.69 (repeated removal of the DSC curve).

На сплаве АМг6 проведены исследования термодинамических закономерностей развитого пластического течения с помощью метода дифференциальной сканирующей калориметрии с целью исследования баланса запасенной энергии, связанной с накоплением дефектов, и диссипативными процессами, ассоциируемыми с пластическим течением. Переход через первую критическую точку соответствует формированию множественных областей локализованной пластичности и увеличением запасённой энергии в ходе деформации, что подтверждается увеличением доли запасённой энергии и наблюдением рельефа, вызванного появлением полос скольжения (Рис. 2b), на образце с деформацией 0.35. Переход через вторую критическую точку связывается с формированием очагов макроскопического разрушения (Рис. 2c) с интенсивным перераспределением запасённой энергии деформирования, что проявляется в виде уменьшения доли запасённой энергии для образца с деформацией 0.69 .

Благодарности/Acknowledgements. Работа выполнена при финансовой поддержке РФФИ (грант 17-01-00867_a, 19-48-590016_p_a). / This work has been carried out with the financial support of the Russian Foundation for Basic Research Ggrant Nr.17-01-00867_a, 19-48-590016_p_a).

\section{Литература/References}

1. G. D’Anna, F. Nori. Phys. Rev. Lett. 85 (19), 4096 (2000). $\underline{\text { Crossref }}$

2. I. Panteleev, C. Froustey, O. Naimark. Comp. Contin. Mech. 2 (3), 70 (2009). (in Russian) [И.А. Пантелеев, C. Froustey, О.Б. Наймарк. Вычислительная механика сплошных сред. 2 (3), 70 (2009).] Crossref

3. T. Tretyakova, V. Wildemann. Physical Mesomechanics. 20 (2), 71 (2017). (in Russian) [T.В. Третьякова, В.Э. Вильдеман. Физическая Мезомеханика. 20 (2), 71 (2017).] 
4. L. Zuev, V. Danolov, B. Semukhin. Progress in Physics of Metals. 3, 237 (2002). (in Russian) [Л.Б. Зуев, В. И. Данилов, Б. С. Семухин. Успехи физ. мет. 3, 237 (2002).] Crossref

5. L. Zuev. Physical mesomechanics. 14 (3), 85 (2011). (in Russian) [Л.Б. Зуев. Физическая мезомеханика. 14 (3), 85 (2011).]

6. M. Zaiser, E. Aifantis. International Journal of Plasticity. 22 (8), 1432 (2006). Crossref

7. M. Zaiser, F. M. Grasset, V. Koutsos, E.C. Aifantis. Phys. Rev. Lett. 93 (19), 195507 (2004). Crossref

8. G. Ananthakrishna, S. J. Noronha, C. Fressengeas, L. P. Kubin. Phys. Rev. E. 60 (5), 5455 (1999). Crossref

9. L.P. Kubin, G. Ananthakrishna, C. Fressengeas. Phys. Rev. E. 65 (1), 053501 (2002). Crossref

10. M. Zaiser. Advances in Physics. 55 (1-2), 185 (2006). Crossref

11. D. Efremov, V. Oborin, S. Uvarov, O. Naimark. PNRPU Mechanics Bulletin. 4, 28 (2017). (in
Russian) [Д.В. Ефремов, В.А. Оборин, С.В. Уваров, О.Б. Наймарк. Вестник ПНИПУ. Механика. (4), 28 (2017).] Crossref

12. J.-F. Pinton, P.C.W. Holdsworth, R. Labbe. Physical Review E. 60 (3), 2452 (1999). Crossref

13. N. Mordant, J.-F. Pinton, F. Chilla. J. Phys. II France. 7, 1729 (1997). Crossref

14. E. K.H. Salje, A. Saxena, A. Planes. In: Avalanches in Functional Materials and Geophysics. Springer, Switzerland (2017) 298 p. $\underline{\text { Crossref }}$

15. G. W.H. Hohne, W. F. Hemminger, H.-J. Flammersheim. In: Differential Scanning Calorimetry. Springer, Berlin (2003) 298 p. $\underline{\text { Crossref }}$

16. N. J. Luiggi, M. Valera, J. P. Rodriguez, J. Prin. Journal of Metallurgy. 2014, 345945 (2014). Crossref

17. O. Naimark. Physical Mesomechanics. 18 (3), 71 (2015).

18. O. B. Naimark. Physical Mesomechanics. 13 (5-6), 306 (2010). Crossref

19. R. Labbe, J.-F. Pinton, S. Fauve. J. Phys. II France. 6, 1099 (1996). rossref 